\title{
Long non-coding RNA SNHG20 promotes ovarian cancer development by targeting microRNA-338-3p to regulate MCL1 expression
}

\author{
DING WANG, ZHIYING LI, HUI LI, JIAO LU and QI QIN \\ Department of Gynecology, The Affiliated Renhe Hospital of China Three Gorges University, \\ Yichang, Hubei 443001, P.R. China
}

Received May 13, 2020; Accepted November 4, 2020

DOI: $10.3892 / \mathrm{ol} .2020 .12391$

\begin{abstract}
Long non-coding RNAs (lncRNAs) and microRNAs (miRNAs/miRs) were reported to be associated with the development of ovarian cancer (OC). Increasing evidence demonstrated that lncRNA SNHG20 and miR-338-3p were involved in OC. However, the functional mechanism of lncRNA SNHG20 and miR-338-3p in OC development remains unknown. The expression of SNHG20, miR-338-3p and myeloid cell leukemia 1 (MCL1) was detected by reverse transcription-quantitative PCR. MTT assay, flow cytometry and transwell migration and invasion assays were used to assess cell proliferation, apoptosis, migration and invasion, respectively. The relative protein expression was detected by western blot analysis. The interaction between miR-338-3p and SNHG20 or MCL1 was predicted by starBase v3.0, and subsequently confirmed by dual-luciferase reporter assay. Besides, mouse xenograft assay was carried out to explore the effect of SNHG20 on tumor growth in vivo. The levels of SNHG20 and MCL1 were upregulated, while miR-338-3p level was downregulated in OC tissues and cells. SNHG20 knockdown repressed OC cell proliferation, migration, invasion and epithelial-mesenchymal transition, and induced apoptosis. Interestingly, SNHG20 targeted miR-338-3p to regulate MCL1 expression. miR-338-3p depletion or MCL1 overexpression could reverse the effects of SNHG20 knockdown on OC cells. Besides, SNHG20 knockdown impeded tumor growth in vivo. In conclusion, the present study demonstrated that SNHG20 regulates OC development via modulation of the miR-338-3p/MCL1 axis, providing the theoretical basis for the treatment of OC.
\end{abstract}

Correspondence to: Dr Ding Wang, Department of Gynecology, The Affiliated Renhe Hospital of China Three Gorges University, 410 Yiling Avenue, Yichang, Hubei 443001, P.R. China E-mail: opdsfl@163.com

Key words: ovarian cancer, SNHG20, microRNA-338-3p, MCL1

\section{Introduction}

Ovarian cancer (OC), a common gynecological tumor, is the fifth leading cause of tumor-associated death in women, and there were $\sim 22,240$ new cases and $\sim 14,070$ deaths associated with OC every year worldwide (1). Most patients with OC were diagnosed at the advanced stage with tumor metastasis and recurrence (2). Despite achieving great progress in the technologies for the detection and therapy of OC, the overall survival rate of patients with OC remains low. Therefore, it is urgent to find the effective therapeutic targets for OC.

Long non-coding RNAs (IncRNAs), with over 200 nucleotides, are a group of conserved RNAs that play pivotal function in cancer progression, such as cell proliferation, mobility, apoptosis and autophagy (3-6). In recent years, lncRNAs were reported to be involved in various human cancer types, including OC $(7,8)$. Small nucleolar RNA host gene 20 (SNHG20), 8,275 bases in length, was originally identified as an oncogene in hepatocellular cancer (9). A previous study indicated that SNHG20 knockdown suppressed the growth of OC cells through modulating the levels of downstream genes (10). However, the functional mechanism of SNHG20 is largely unknown in OC.

MicroRNAs (miRNAs/miRs), 22 nucleotides, exert crucial roles via inhibiting translation or promoting degradation $(11,12)$. Multiple studies demonstrated that miRNAs participated in the progression of various cancer types, including lung cancer (13), osteosarcoma (14), thyroid cancer (15) and OC (16). A recent study suggested that miR-338-3p level was decreased in OC, and upregulation of miR-338-3p repressed OC cell proliferation (17). Moreover, Liu et al (18) demonstrated that miR-338-3p acted as a tumor suppressor to regulate cell proliferation, mobility and apoptosis in OC. These data revealed that miR-338-3p played an important role in OC. Thus, the present explored the underlying mechanism of miR-338-3p in OC.

Myeloid cell leukemia 1 (MCL1) belongs to B-cell lymphoma 2 (BCL-2) family, which is considered as a class of cell apoptosis regulators in human cancer (19). It was reported that many factors, including deubiquitinase and miRNAs, mediated the level of MCL1 to affect cell growth in OC. For instance, USP13 regulated MCL stabilization to modulate 
tumor growth (20). Su et al (21) demonstrated that miR-142-5p mediated cisplatin-induced cell apoptosis via regulating MCL expression. Thus, MCL pathway is associated with OC development. Therefore, it is important to examine the function of MCL in OC cells.

Here, we determined the levels of SNHG20, miR-338-3p and MCL1 in OC tissues and cells. Furthermore, the function of SNHG20 was investigated in OC in vitro and in vivo. Besides, the underlying mechanism of SNHG20 in OC progression was explored.

\section{Materials and methods}

Tissues and cell culture. 30 OC tissues and 30 adjacent normal tissues were obtained from the patients at the hospital of The Affiliated Renhe Hospital of China Three Gorges University from April 2016 to January 2019. The average age of OC patients was $(52.02 \pm 10.88)$ years, in which there were 18 patients $\geq 50$ years and 12 patients $<50$ years. These tissue samples were stored at $-80^{\circ} \mathrm{C}$ until use. This research was approved by the Ethics Review Committees of The Affiliated Renhe Hospital of China Three Gorges University. All participates provided written informed consent.

Normal human ovarian surface epithelial (HOSE) cell line was provided by ScienCell Research Laboratories, Inc. OC cell lines (OVCAR3, SKOV3, A2780 and CAOV-3) were bought from the Cell Bank of Type Culture Collection of the Chinese Academy of Sciences. These cells were cultured in RPMI-1640 medium (cat. no. 22400089; Invitrogen; Thermo Fisher Scientific, Inc.) containing $10 \%$ fetal bovine serum (FBS; cat. no. SH30068.03; Hyclone; Cytvia) and 1\% penicillin/streptomycin (cat. no. 516106; EMD Millipore) in a humidified incubator at $37^{\circ} \mathrm{C}$ with $5 \% \mathrm{CO}_{2}$.

RNA extraction and reverse transcription-quantitative $(R T-q) P C R$. Total RNA was isolated from OC tissues or cells using TRIzol reagents (Invitrogen; Thermo Fisher Scientific, Inc.), and subsequently used to synthesize complementary DNA using the SuperScript III ${ }^{\circledR}$ (cat. no. 18080-044; Invitrogen; Thermo Fisher Scientific, Inc.) based on the recommended protocol $\left(65^{\circ} \mathrm{C}\right.$ for $5 \mathrm{~min}, 50^{\circ} \mathrm{C}$ for $30-60 \mathrm{~min}$ and $70^{\circ} \mathrm{C}$ for $\left.15 \mathrm{~min}\right)$. Subsequently, an SYBR Premix ExTaq kit (cat. no. RR047A; Takara Bio, Inc.) was applied to perform $\mathrm{qPCR}$. The thermocycling conditions were as follows: $94^{\circ} \mathrm{C}$ for $5 \mathrm{~min}$, followed by 40 cycles of $94^{\circ} \mathrm{C}$ for $30 \mathrm{sec}, 60^{\circ} \mathrm{C}$ for $1 \mathrm{~min}$, and $72^{\circ} \mathrm{C}$ for $30 \mathrm{sec}$. The data were normalized by U6 or GAPDH level, and analyzed using the $2^{-\Delta \Delta \mathrm{Cq}}$ method (22). The primers used in the study are listed in Table I.

Cell transfection. Small interfering RNA against SNHG20 (si-SNHG20, final concentration, $40 \mathrm{nM}$ ), small hairpin RNA against SNHG20 (sh-SNHG20, final concentration, $1 \mu \mathrm{g} / \mathrm{ml}$ ), miR-338-3p mimic (miR-338-3p, final concentration, $20 \mathrm{nM}$ ), miR-338-3p inhibitor (anti-miR-338-3p, final concentration, $25 \mathrm{nM}$ ), and the relative negative controls (si-NC, final concentration, $40 \mathrm{nM}$; sh-NC, final concentration, $1 \mu \mathrm{g} / \mathrm{ml}$; miR-NC, final concentration, $20 \mathrm{nM}$; and anti-miR-NC, final concentration, $25 \mathrm{nM}$ ) were purchased from Shanghai GenePharma Co. Ltd. (cat. no. B03001). For
Table I. Reverse transcription-quantitative PCR primer sequences.

\begin{tabular}{ll} 
Gene & \multicolumn{1}{c}{ Sequence } \\
\hline SNHG20 & F: 5'-ATGGCTATAAATAGATACACGC-3' \\
& R: 5'-GGTACAAACAGGGAGGGA-3' \\
miR-338-3p & F: 5'-AACCGGTCCAGCATCAGTGATT-3' \\
& R: 5'-CAGTGCAGGGTCCGAGGT-3' \\
MCL1 & F: 5'-GGGCAGGATTGTGACTCTCATT-3' \\
& R: 5'-GATGCAGCTTTCTTGGTTTATGG-3' \\
U6 & F: 5'-TGCGGGTGCTCGCTTCGGCAGC-3' \\
& R: 5'-CCAGTGCAGGGTCCGAGGT-3' \\
GAPDH & F: 5'-ATCACTGCCACCCAGAAGAC-3' \\
& R: 5'-TTTCTAGACGGCAGGTCAGG-3'
\end{tabular}

SNHG20, small nucleolar RNA host gene 20; MCL1, myeloid cell leukemia 1; U6, U6 small nuclear 1; F, forward; R, reverse.

Table II. Sequences of constructs used for transfection.

\begin{tabular}{ll}
\hline Gene & \multicolumn{1}{c}{ Sequence (5'-3') } \\
\hline si-SNHG20 & GCCACUCACAAGAGUGUAUTT \\
si-NC & GGATACGGAGTACTATAGC \\
sh-SNHG20 & CACCGGCCCAGATTGGTACATTTC \\
& GAAAAATGTACCAATCTGG \\
miR-338-3p & UUUGAGCAGCACUCAUUUUUGC \\
miR-NC & CAGUACUUUUAGUGUGUACAA \\
anti-miR-338-3p & CAACAAAAUCACUGAUGCUGGA \\
anti-miR-NC & UUGUAAGUUGCGACAGCCACUCA \\
\hline
\end{tabular}

Si, small interference RNA; SNHG20, small nucleolar RNA host gene 20; NC, negative control; sh, short hairpin RNA; miR-338-3p, miRNA-338-3p mimic; anti-miR-338-3p, miRNA-338-3p inhibitor; NC-negative control.

overexpression of SNHG20 or MCL1 (pcDNA-SNHG20, final concentration, $0.8 \mu \mathrm{g} / \mathrm{ml}$; pcDNA-MCL1, final concentration, $1 \mu \mathrm{g} / \mathrm{ml})$, its sequence was inserted into the pcDNA3.1 vector (cat. no. GM-1013P001-P10; GenePharma). The sequences of the constructs are listed in Table II and Data S1. Cell transfection was carried out using Lipofectamine ${ }^{\circledR} 3000$ (cat. no. L3000-015; Invitrogen; Thermo Fisher Scientific, Inc.) in serum free Opti-MEM medium (cat. no. 31985062; Thermo Fisher Scientific, Inc.) and incubated in a humidified incubator at $37^{\circ} \mathrm{C}$ with $5 \% \mathrm{CO}_{2}$ for $6 \mathrm{~h}$, followed by medium being changed to RPMI-1640 medium containing $10 \%$ fetal bovine serum and $1 \%$ penicillin/streptomycin. Subsequent experimentation was performed 24 or $48 \mathrm{~h}$ post-transfection.

Cell proliferation assay. The MTT kit (M5655; Sigma-Aldrich; Merck KGaA) was used to examine cell proliferation according to the manufacturer's manual. Briefly, SKOV3 and A2780 cells were transfected for $48 \mathrm{~h}$, and then cultured for 24,48 or $72 \mathrm{~h}$. 
A

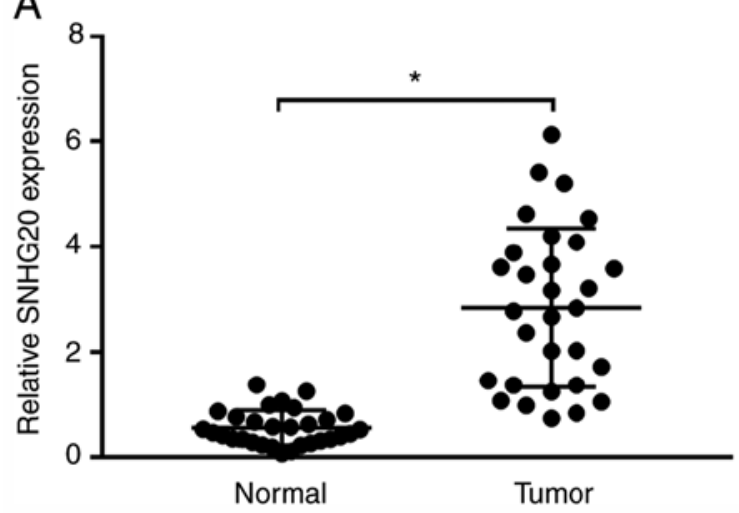

B

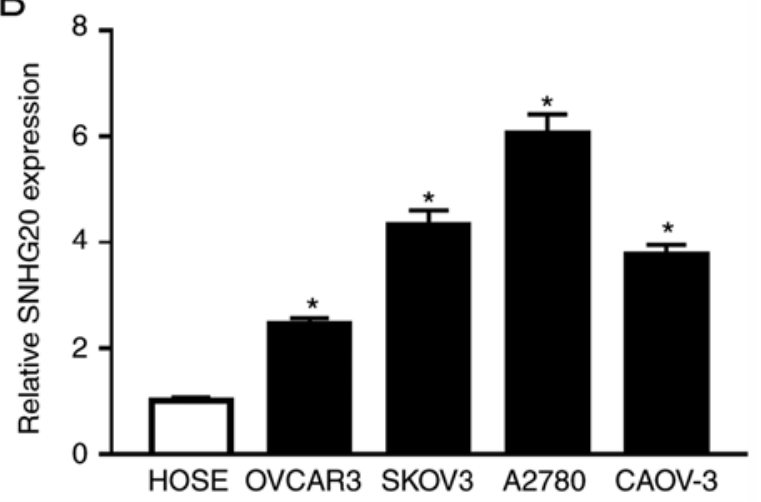

Figure 1. SNHG20 expression level is higher in OC tissues and cells. Expression level of SNHG20 was detected by reverse transcription-quantitative PCR assay in OC tissues and paired adjacent normal tissues (A) as well as in OC cells and human ovarian surface epithelial cells (B) * $<<0.05$. SNHG20, small nucleolar RNA host gene 20; OC, ovarian cancer.

Next, $10 \mu \mathrm{l}$ MTT solution was added to treat the cells at $37^{\circ} \mathrm{C}$ for $4 \mathrm{~h}$, and then the precipitation was incubated with dimethyl sulfoxide. Finally, the absorbance was determined using the microplate reader (Bio-Rad Laboratories, Inc.) at $490 \mathrm{~nm}$.

Cell apoptosis assay. Cell apoptosis was measured using Annexin V-fluorescein isothiocyanate (FITC)/propidium iodide (PI) Apoptosis Detection kit (cat. no. A211-01/02; Vazyme Biotech Co., Ltd.) according to the manufacturer's manual. Briefly, SKOV3 and A2780 cells were cultured for $48 \mathrm{~h}$. Then, the cells were collected, washed and stained with $5 \mu 1$ Annexin V-FITC and PI. Finally, cell apoptosis was examined using the flow cytometer (BD Biosciences, Inc.) and FlowJo software version v.10.0.6 (Becton, Dickinson and Company).

Western blot assay. Tissue or cell lysates were obtained using lysis buffer (cat. no. P0013B; Beyotime Institute of Biotechnology). A BCA Protein Assay kit was employed to determine the protein concentration. The samples (30 $\mu \mathrm{g} /$ lane) were separated by $10 \%$ dodecyl sulfate, sodium salt-polyacrylamide gel electrophoresis (SDS-PAGE), transferred to polyvinyl difluoride membrane (PVDF), and blocked by $5 \%$ fat-free milk at room temperature. Next, the membranes were incubated at $4{ }^{\circ} \mathrm{C}$ overnight with the primary antibody against MCL1 (1:1,000; cat. no. ab243136; Abcam), B-cell lymphoma 2 (Bcl-2; 1:1,000; cat. no. ab32124; Abcam), BCL2 associated X (Bax; 1:1,000; cat. no. ab32503; Abcam), cleaved caspase-3 (c-caspase-3; 1:1,000; ab32042; Abcam), procaspase-3 (1:1,000; cat. no. ab32499; Abcam), vimentin (1:1,000; cat. no. ab227639; Abcam), E-cadherin (1:1,000; cat. no. ab238099; Abcam), N-cadherin (1:1,000; ab76057; Abcam) or GAPDH (1:1,000; cat. no. ab9485; Abcam). Membranes were then incubated with the corresponding secondary antibody goat anti-rabbit IgG H\&L (HRP) (1:2,000; cat. no. ab214880; Abcam) for $1 \mathrm{~h}$ at room temperature. Finally, an ECL detection system (EMD Millipore) was applied to detect the signal, and quantified using ImageJ software V1.8.0 (National Institutes of Health).

Cell migration and invasion assay. Transwell chamber (cat. no. 3450; Corning Life Sciences) was applied to examine cell mobility. For transwell migration assay, transfected SKOV3 and A2780 cells in $100 \mu 1$ RPMI-1640 medium without FBS were seeded into the top chamber. The bottom chamber was filled with $500 \mu 1$ RPMI-1640 medium with $10 \%$ FBS. After $24 \mathrm{~h}$ incubation, the migratory cells on the bottom chamber were counted using an IX91 inverted light microscope (Olympus, Tokyo, Japan) (magnification, x20). For transwell invasion assay, the upper chamber was pre-coated with Matrigel (cat. no. 356234; BD Biosciences), all other following steps were the same as that for the cell migration assay.

Dual luciferase reporter assay. Potential binding sites between miR-338-3p and SNHG20 or MCL1 were predicted using an online bioinformatics database (starBase v.2.0; http://starbase. sysu.edu.cn/). The wide-type or mutant-type of SNHG20 or MCL1 3'untranslated region (UTR) (WT/MUT-SNHG20 or MCL1 3'UTR-WT/MUT) was cloned into the pGL3 vector (cat. no. E1761; Promega Corporations), and co-transfected into SKOV3 and A2780 cells with miR-338-3p or miR-NC. After $48 \mathrm{~h}$ incubation, the luciferase density was measured using the dual luciferase assay system (cat. no. E1910; Promega Corporations). Firefly luciferase activity was normalized to Renilla luciferase activity and the average of triplicate samples was calculated.

Mouse xenografts. Six BALB/c nude mice were randomly divided into the following two groups ( $\mathrm{n}=3$ per group): i) sh-NC group; and ii) sh-SNHG20. The mice (age, 6 weeks) were subcutaneously injected with A2780 cells $\left(1 \times 10^{6}\right)$ transfected with sh-SNHG20 or sh-NC into the left hind back of nude mice. After 7 days, tumor volume (length $\mathrm{x}$ width ${ }^{2} / 2$ ) was calculated every 4 days. After 27 days, the mice were sacrificed by cervical dislocation, following deep anesthesia with $2 \%$ isoflurane, and tumor tissues were collected. Tumor weight was analyzed. This experiment was carried out in line with the guidance of the National Animal Care and Ethics Institution and was authorized by the Animal Research Committee of the Affiliated Renhe Hospital of China Three Gorges University.

Statistical analysis. Each experiment was conducted at least three times independently. Experimental data were presented by mean \pm standard deviation (SD). Two independent groups 
A

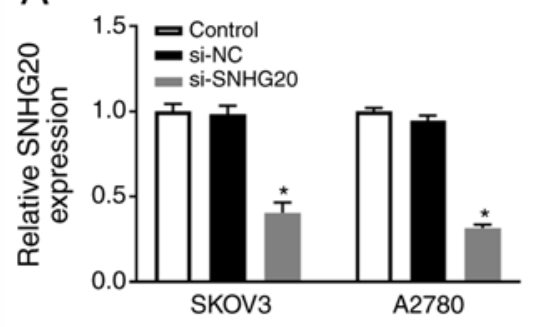

B

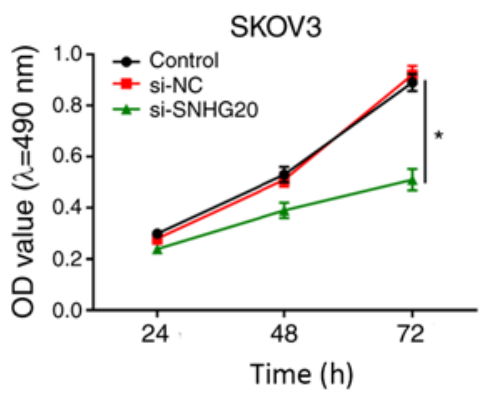

C

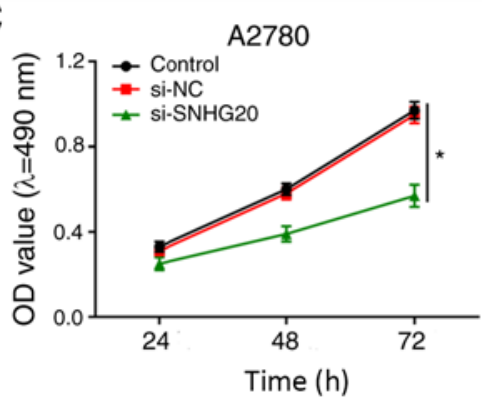

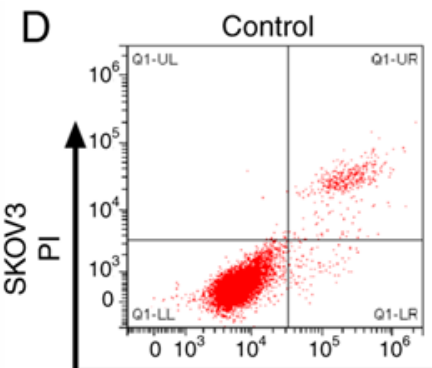
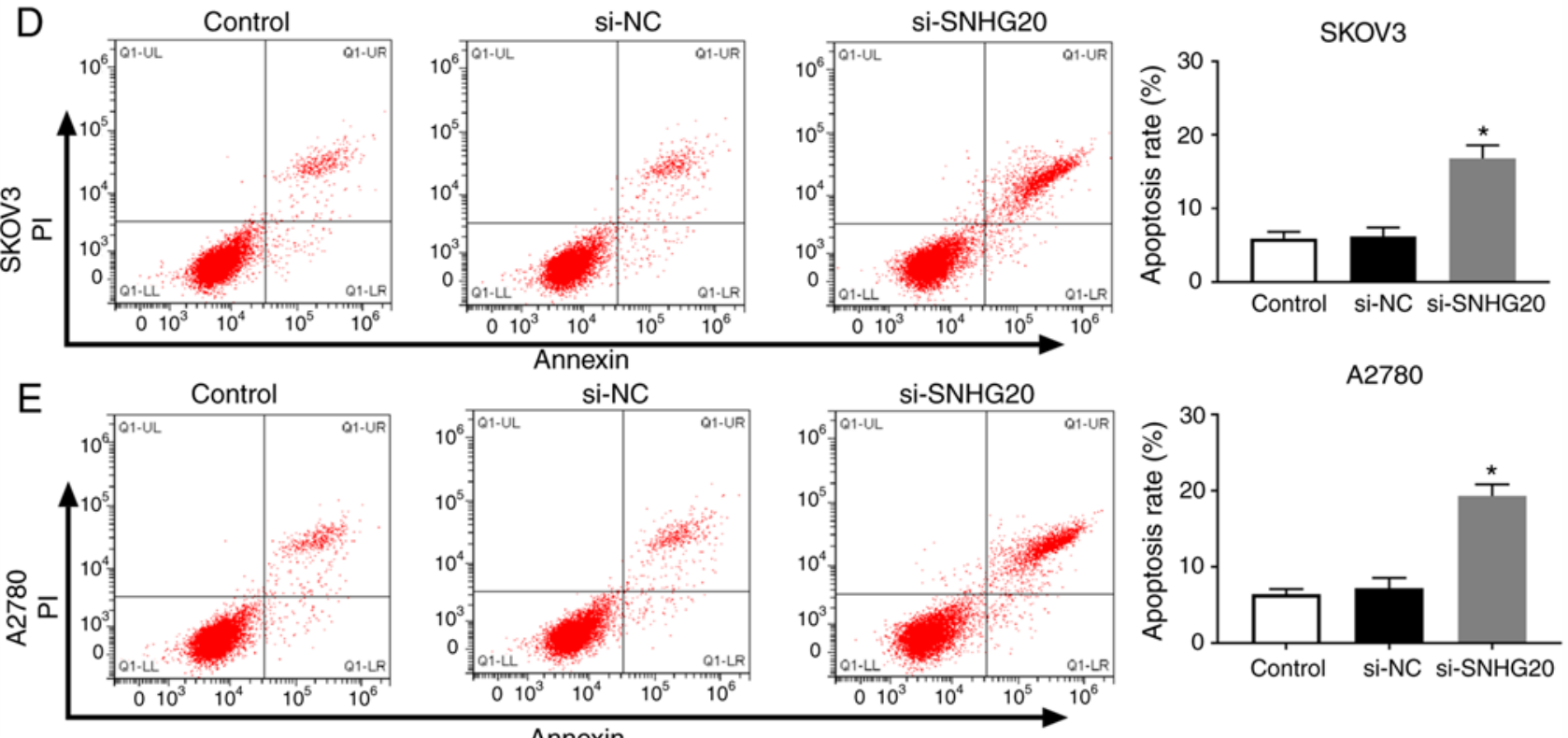

si-
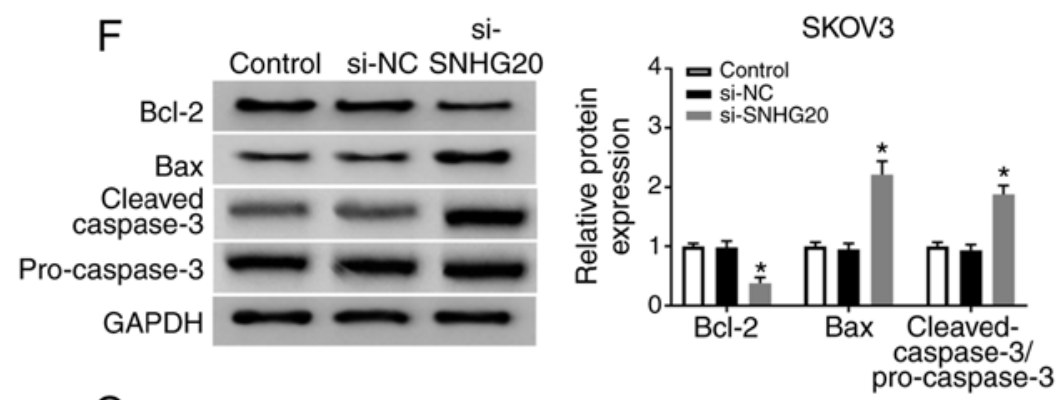

G

si-
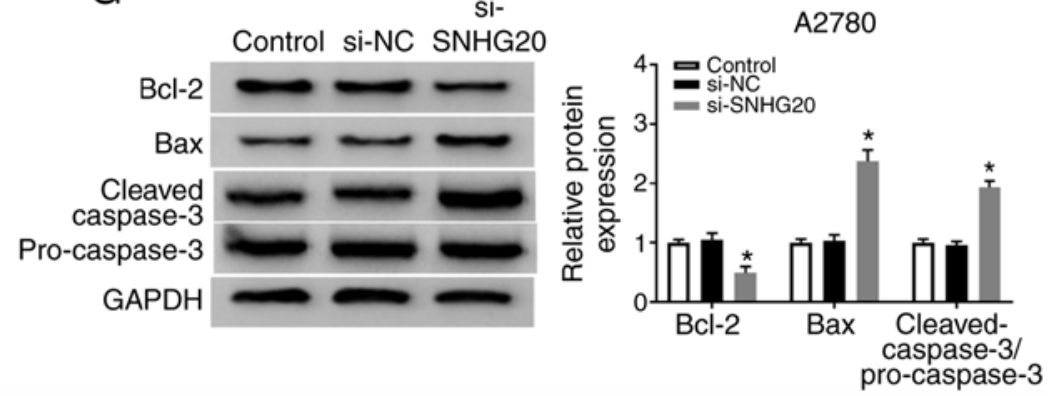

Figure 2. SNHG20 knockdown suppresses OC cell proliferation and promotes apoptosis. (A) Reverse transcription-quantitative PCR was used to detect the expression of SNHG20 in SKOV3 and A2780 cells transfected with si-NC or si-SNHG20. (B and C) MTT assay was performed to assess the proliferation ability of SKOV3 and A2780 cells transfected with si-NC or si-SNHG20. (D and E) Flow cytometry was employed to measure the apoptotic rate of SKOV3 and A2780 cells transfected with si-NC or si-SNHG20. (F and G) Western blot assay was carried out to examine the levels of cell apoptosis-associated proteins. "P<0.05. SNHG20, small nucleolar RNA host gene 20; OC, ovarian cancer; si-NC, small interfering RNA against negative control; si-SNHG20, small interfering RNA against SNHG20.

were analyzed using Student's-t test. ANOVA followed by Tukey's test was used to assess the difference among more than two groups. Spearman's correlation coefficient was calculated to analyze the association between the levels of two genes. $\mathrm{P}<0.05$ was considered to indicate a statistically significant difference. 

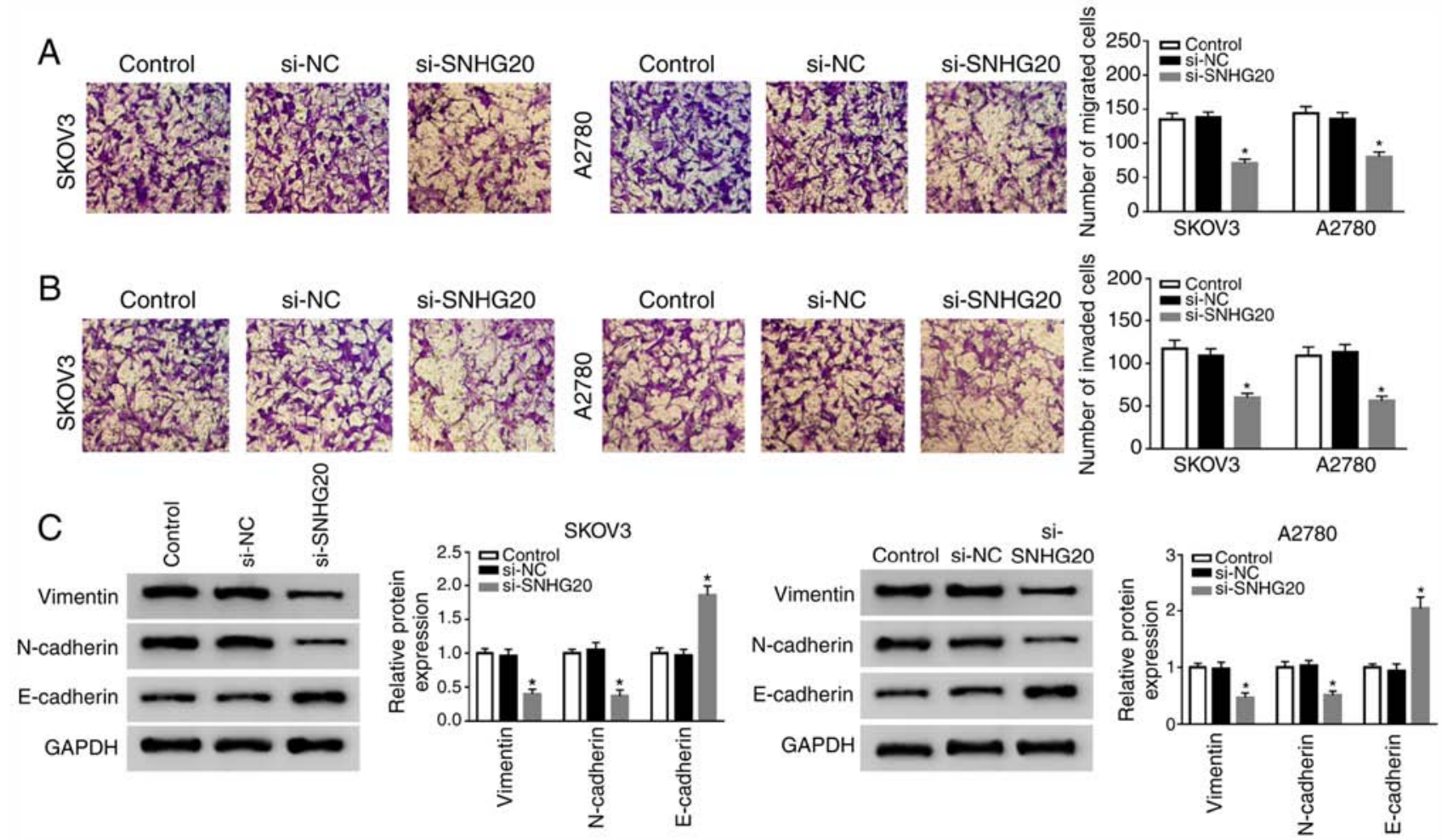

Figure 3. SNHG20 knockdown inhibits the migration, invasion and EMT of OC cells. SKOV3 and A2780 cells were transfected with si-NC or si-SNHG20. (A and B) Cell migratory and invasive abilities were analyzed using transwell assay in SKOV3 and A2780 cells. (C) Relative protein levels of EMT markers were determined by western blot assay. ${ }^{*} \mathrm{P}<0.05$. SNHG20, small nucleolar RNA host gene 20; EMT, epithelial-mesenchymal transition; OC, ovarian cancer; si-NC, small interfering RNA against negative control; si-SNHG20, small interfering RNA against SNHG20.

\section{Results}

Expression of SNHG2O is higher in OC tissues and cells. Firstly, RT-qPCR assay was used to detect the expression of SNHG20 in OC tissues and the adjacent normal tissues. As shown in Fig. 1A, SNHG20 expression was significantly higher in OC tissues. Moreover, SNHG20 level was higher in OC cells (OVCAR3, SKOV3, A2780 and CAOV-3) compared with that in HOSE cells (Fig. 1B). These results indicate that SNHG20 level might be associated with the development of OC.

SNHG2O knockdown represses the proliferation, migration, invasion and EMT, whilst inducing apoptosis in OC cells. To investigate the function of SNHG20 in OC cells, si-SNHG20 was transfected into SKOV3 and A2780 cells to deplete SNHG20 expression. Knockdown efficiency was confirmed by RT-qPCR assay (Fig. 2A). As indicated in Fig. 2B and C, SNHG20 knockdown suppressed cell proliferation notably. Furthermore, flow cytometry analysis demonstrated that cell apoptosis was significantly induced by the downregulation of SNHG20 in SKOV3 and A2780 cells (Fig. 2D and E). Meanwhile, the levels of three apoptosis-associated proteins (Bcl-2, Bax, and c-caspase-3) were detected and found that $\mathrm{Bcl}-2$ level was downregulated, while Bax and c-caspase3 levels were upregulated by SNHG20 knockdown (Fig. 2F and G). Next, the effect of SNHG20 on cell mobility was explored. The data suggested that SNHG20 knockdown suppressed cell migration and invasion (Fig. 3A and B). Furthermore, the decreased levels of vimentin and $\mathrm{N}$-cadherin, as well as the increased E-cadherin level, were observed in SNHG20-depleted SKOV3 and A2780 cells (Fig. 3C). Taken together, SNHG20 knockdown inhibited OC development.

$m i R-338-3 p$ is a target of SNHG20. Bioinformatics tool starBase v2.0 predicted that miR-338-3p was a potential target gene of SNHG20 (Fig. 4A). Subsequently, dual-luciferase reporter assay was performed to verify the association between miR-338-3p and SNHG20. The results suggested that miR-338-3p decreased the luciferase activity of WT-SNHG20, whilst having no effect on the luciferase activity of MUT-SNHG20 (Fig. 4B and C), revealing that SNHG20 could bind to miR-338-3p. Successful overexpression efficiency of SNHG20 is demonstrated by Fig. 4D. Moreover, miR-338-3p expression was significantly upregulated by SNHG20 depletion and downregulated by SNHG20 overexpression (Fig. 4E). The present study data showed that miR-338-3p level was decreased in OC tissues and cells (Fig. 4F and G). Furthermore, miR-338-3p level was negatively correlated with SNHG20 level in OC tissues (Fig. 4H). These data suggest that SNHG20 targeted miR-338-3p and downregulated miR-338-3p expression.

SNHG20 depletion suppresses OC progression by targeting $m i R-338-3 p$. To investigate whether SNHG20 affected OC cell progression via suppressing miR-338-3p expression in OC cells, SKOV3 and A2780 cells were transfected with si-NC, si-SNHG20, si-SNHG20 + anti-miR-NC, or si-SNHG20 + anti-miR-338-3p, respectively. Firstly, the data showed that transfection of anti-miR-338-3p dramatically 
A

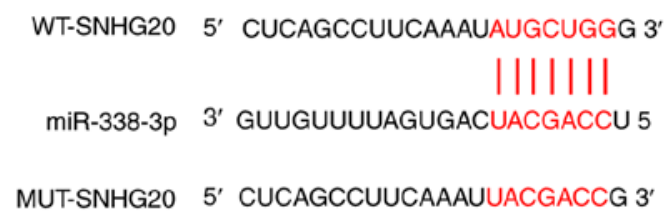

C

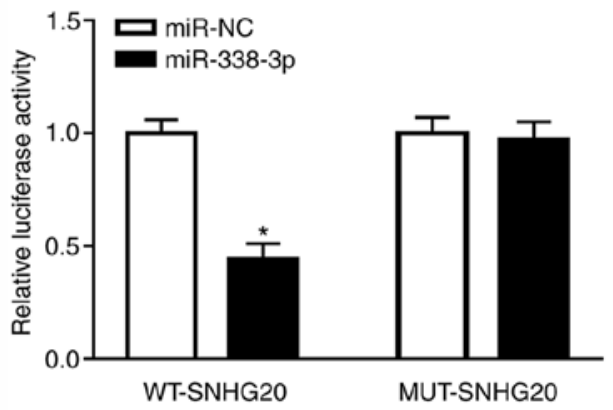

E

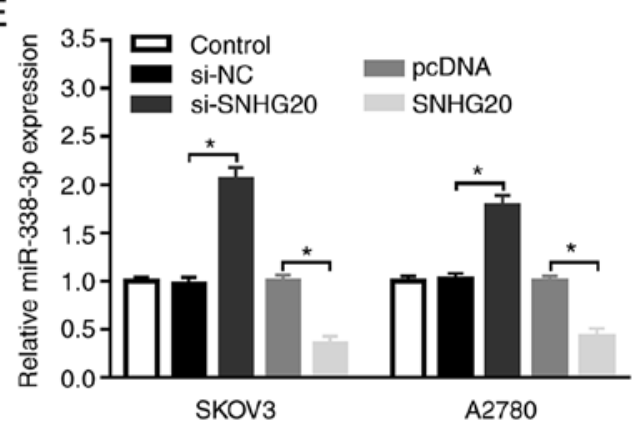

G

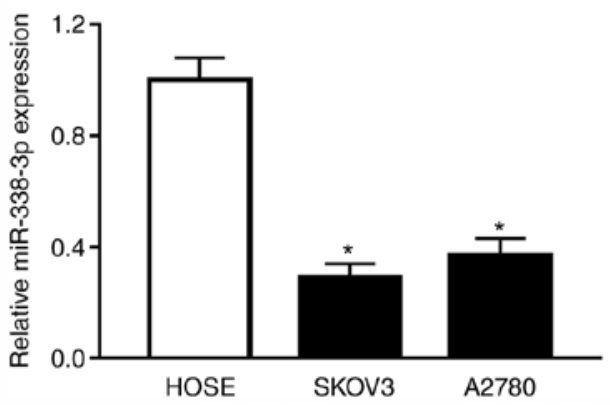

SKOV3

B

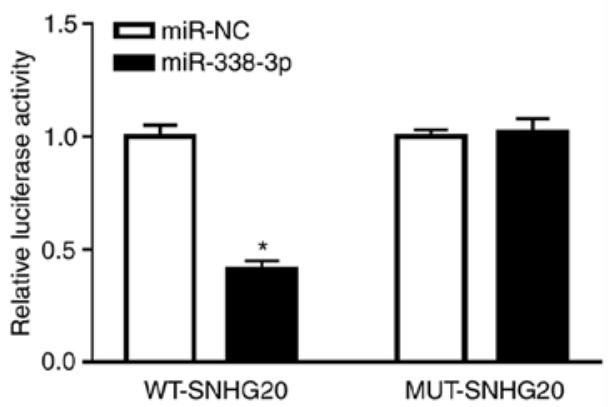

D

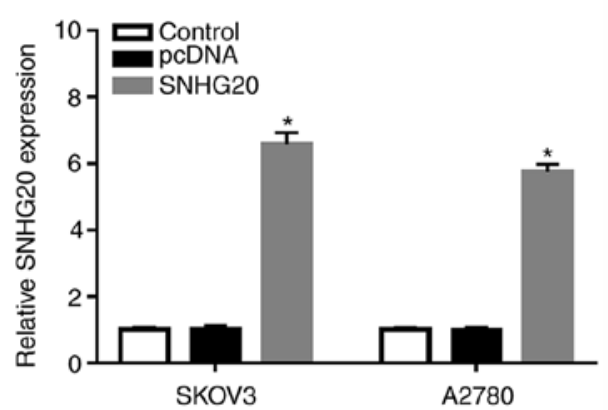

$\mathrm{F}$

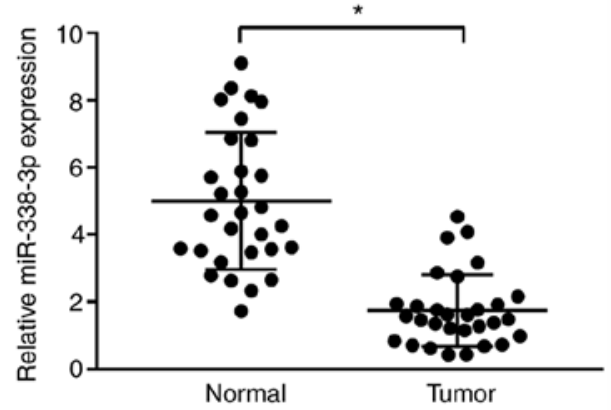

$\mathrm{H}$

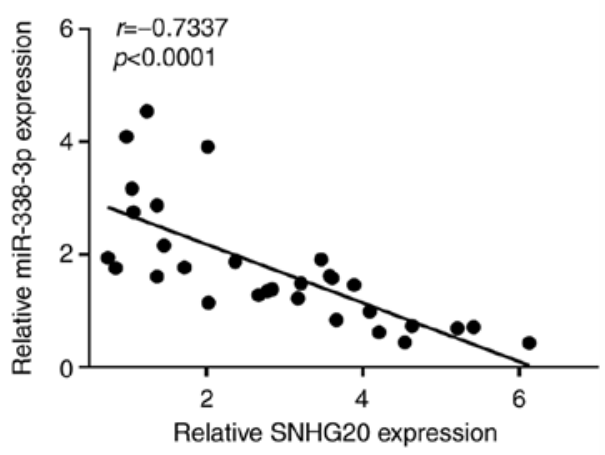

Figure 4. SNHG20 targets miR-338-3p. (A) The interaction between SNHG20 and miR-338-3p was predicted by starBase v2.0. Mutated sites were indicated by the red color. (B and C) Dual-luciferase reporter assay was used to detect the luciferase activity of SKOV3 and A2780 cells co-transfected with miR-NC or miR-338-3p and WT-SNHG20 or MUT-SNHG20. (D) SNHG20 level in SKOV3 and A2780 cells transfected with pcDNA or SNHG20 was detected by RT-qPCR. (E) RT-qPCR was used to measure miR-338-3p expression in SKOV3 and A2780 cells transfected with si-NC, si-SNHG20, pcDNA, or SNHG20, respectively. (F and G) miR-338-3p level was detected by RT-qPCR in OC tissues and the adjacent normal tissues (F) as well as OC cells and human ovarian surface epithelial cells $(\mathrm{G})$. (H) The correlation between SNHG20 and miR-338-3p was analyzed using linear regression. ${ }^{*} \mathrm{P}<0.05$. SNHG20, small nucleolar RNA host gene 20; WT, wild type; MUT, mutant type; miR-NC, miR-negative control mimic; pcDNA, pcDNA3.1 vector; si-NC, small interfering RNA against negative control; si-SNHG20, small interfering RNA against SNHG20; RT-qPCR, reverse transcription-quantitative PCR; OC, ovarian cancer.

downregulated the expression of miR-338-3p, while it was upregulated by miR-338-3p mimic transfection (Fig. 5A). MTT assay suggested that SNHG20 knockdown suppressed cell proliferation, which was reversed by downregulation of miR-338-3p (Fig. 5B and C). In addition, it was found that cell apoptosis induced by SNHG20 knockdown was rescued 
A

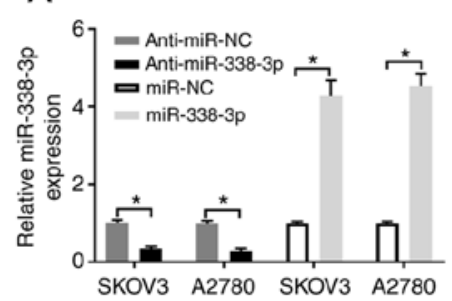

D
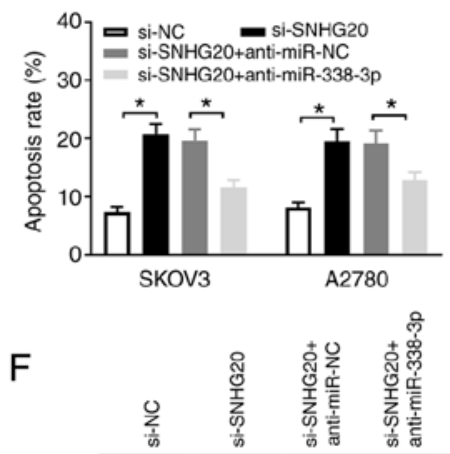

$\mathrm{Bcl}-2$

Bax

Cleaved caspase-3

Pro-caspase-3

GAPDH
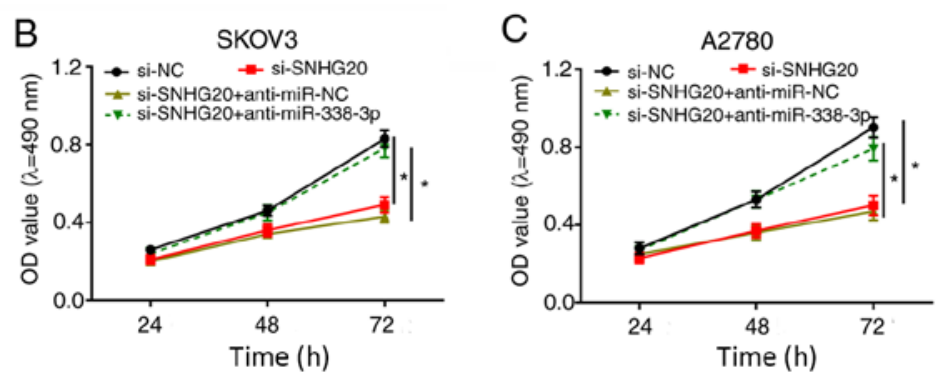

E
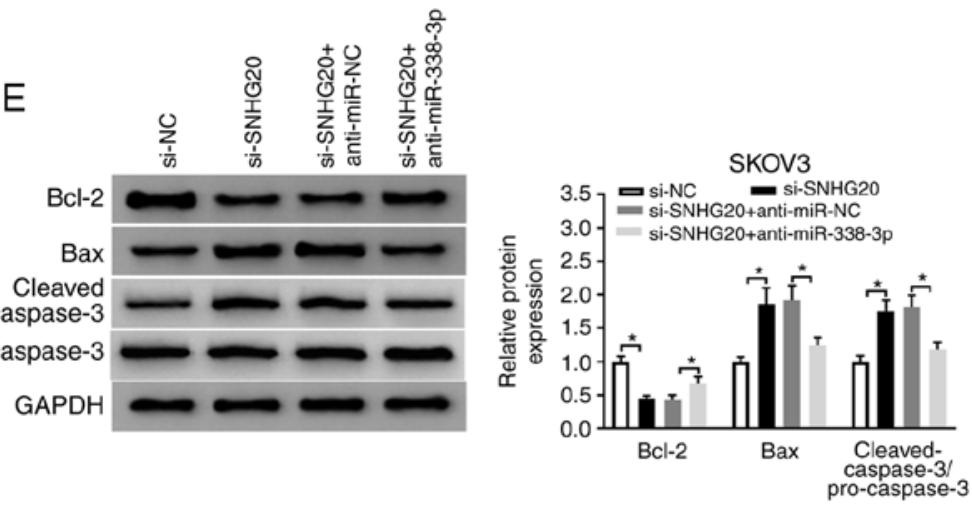

G
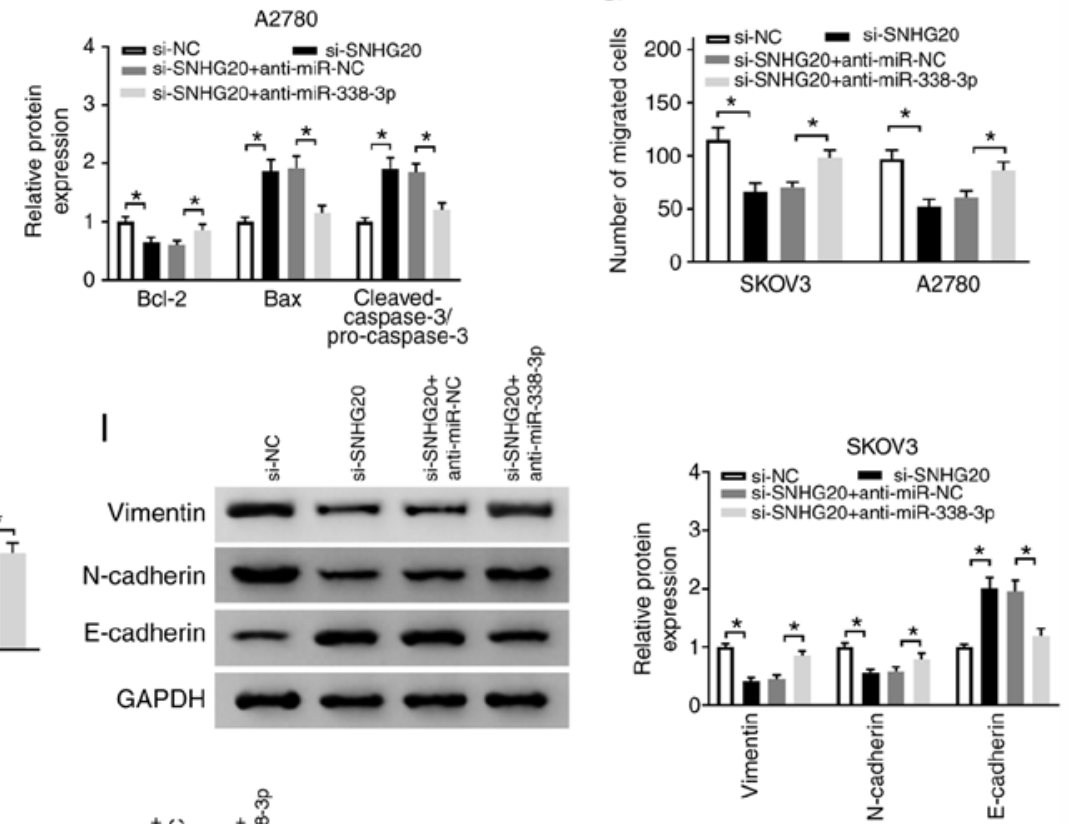
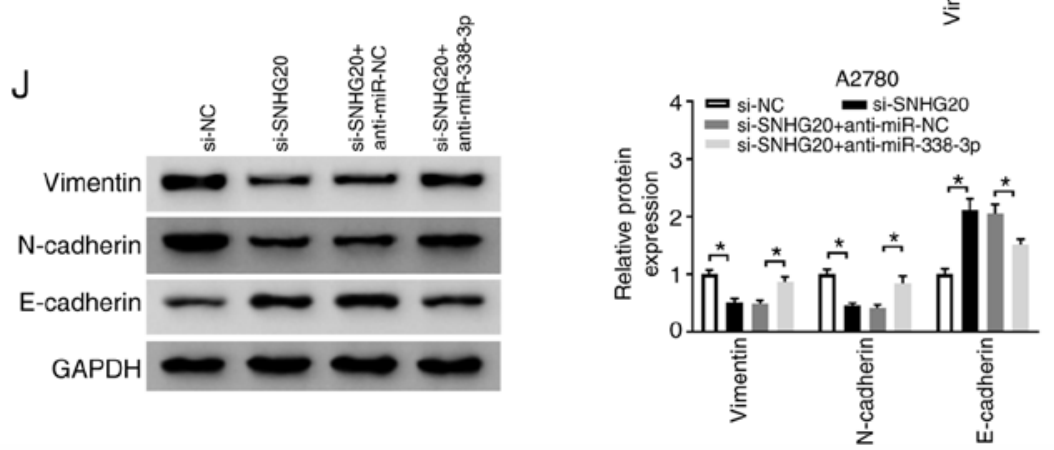

Figure 5. SNHG20 regulates OC progression through sponging miR-338-3p. (A) miR-338-3p level was investigated using reverse transcription-quantitative PCR in SKOV3 and A2780 cells transfected with miR-338-3p or anti-miR-338-3p. (B-J) SKOV3 and A2780 cells were transfected with si-NC, si-SNHG20, si-SNHG20 + anti-miR-NC, or si-SNHG20 + anti-miR-338-3p, respectively. (B and C) MTT assay was employed to measure cell proliferation ability. (D) Cell apoptosis rate was analyzed using flow cytometry. (E and F) The levels of cell apoptosis-associated proteins were determined by western blot assay. $(\mathrm{G}$ and $\mathrm{H})$ Cell migration and invasive abilities were assessed by transwell assay. (I and J) Western blot assay was used to detect the relative protein levels of EMT markers. "P<0.05. SNHG20, small nucleolar RNA host gene 20; OC, ovarian cancer; anti-miR-NC, miR-negative control inhibitor; si-NC, small interfering RNA against negative control; si-SNHG20, small interfering RNA against SNHG20; anti-miR-338-3p, miR-338-3p inhibitor; EMT, epithelial-mesenchymal transition. 
A

MCL1 3' UTR-WT 5' GCACUGUGAAACCCCUGCUGGA 3' miR-338-3p 3' GUUGUUUUAGUGACUACGACCU 5 MCL1 3' UTR-MUT 5' GCACUGUGAAACCCCACGACCA 3'
$\mathrm{B}$

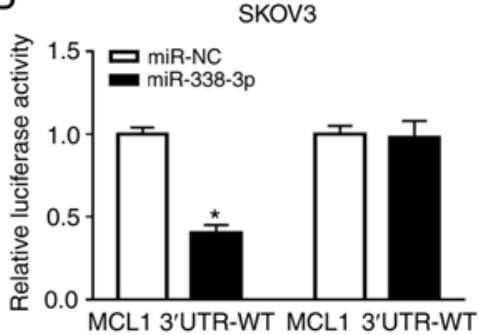

C

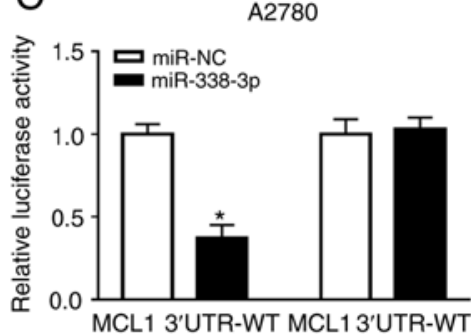

D

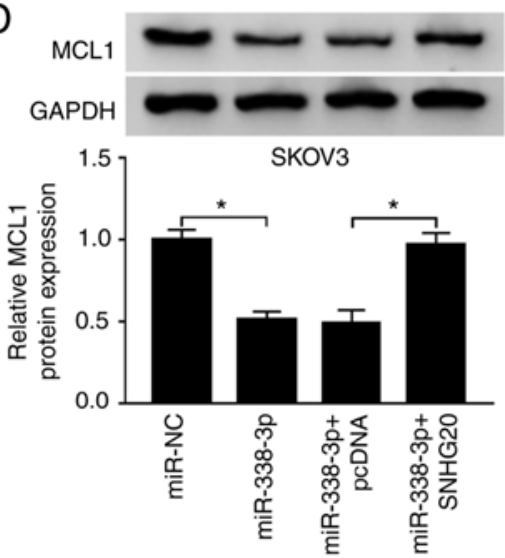

$E$
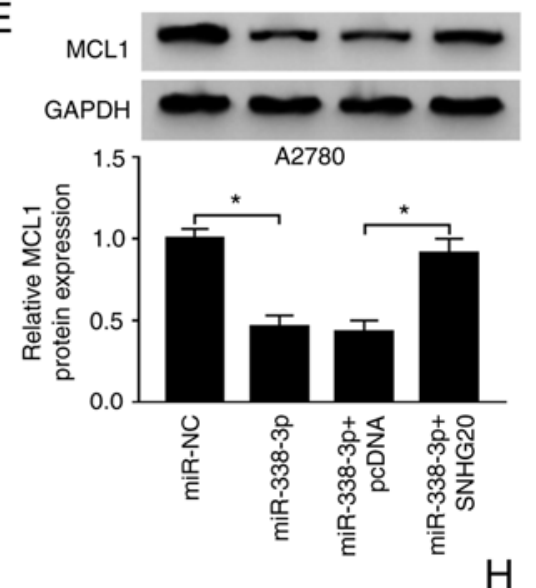

$\mathrm{F}$

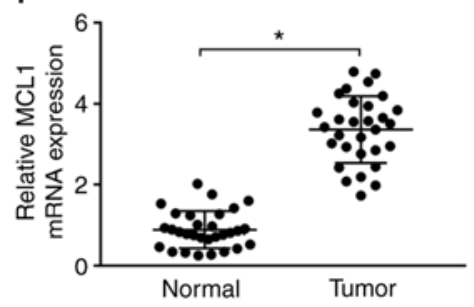

G
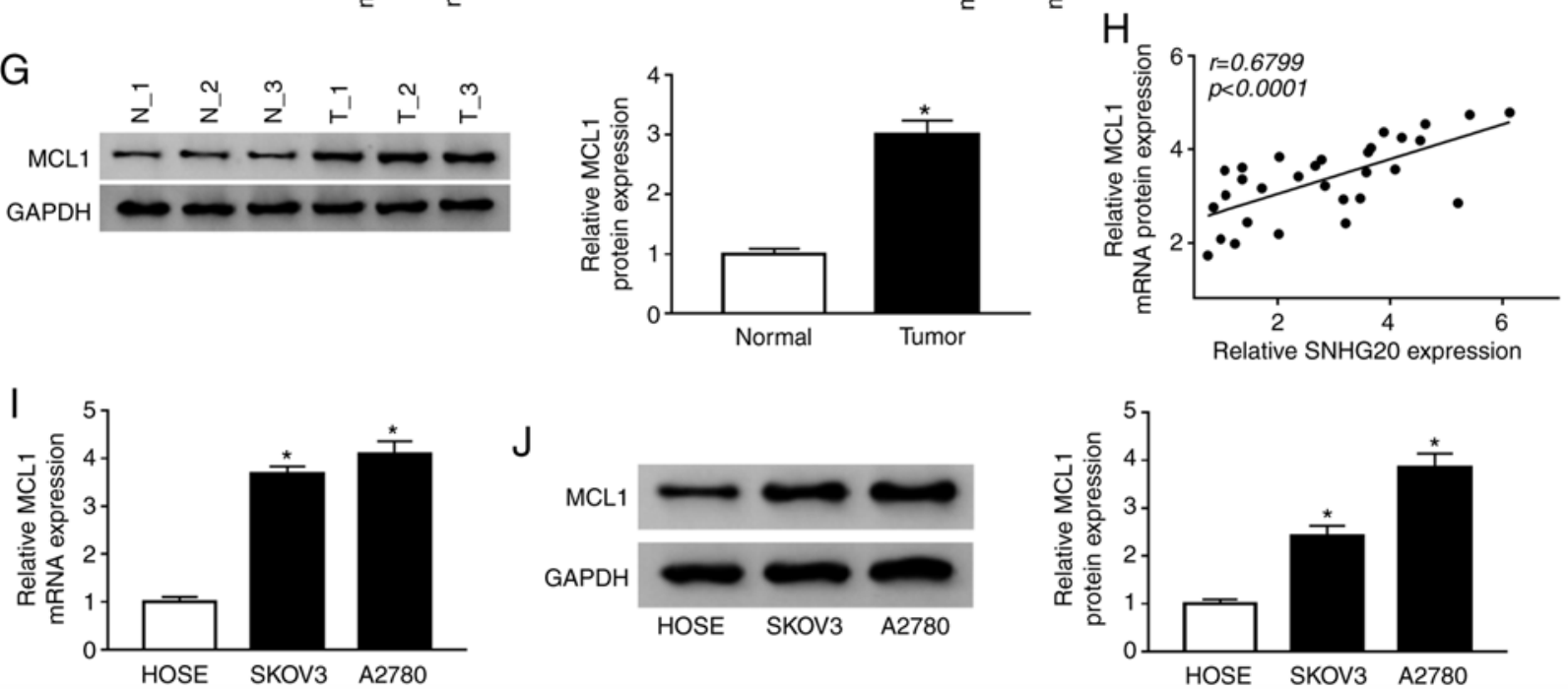

J
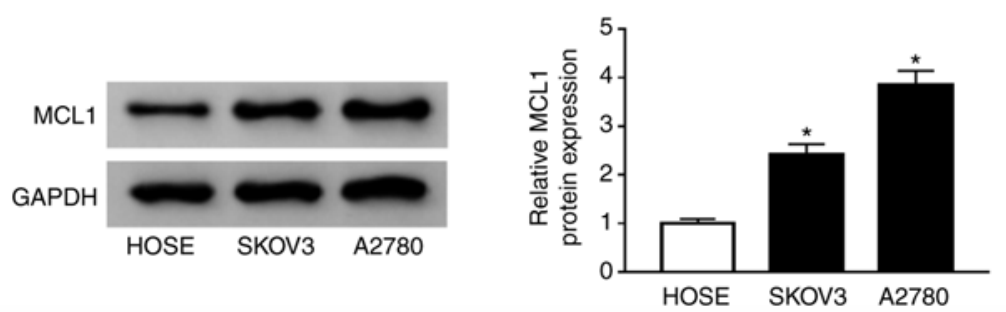

Figure 6. SNHG20 regulates miR-338-3p expression to modulate MCL1 level. (A) The interaction between miR-338-3p and MCL1 was predicted by starBase v2.0. Mutated sites were indicated by the red color. (B and C) The luciferase activity of SKOV3 and A2780 cells co-transfected with MCL1 3'UTR-WT or MCL1 3'UTR-MUT and miR-NC or miR-338-3p was examined by dual-luciferase reporter assay. (D and E) MCL1 expression was measured by western blot analysis in SKOV3 and A2780 cells transfected with miR-NC, miR-338-3p, miR-338-3p + pcDNA or miR-338-3p + SNHG20, respectively. (F and G) Relative mRNA level and protein level of MCL1 were detected by RT-qPCR and western blotting in OC tissues and the adjacent normal tissues. (H) The association between MCL1 level and SNHG20 level was analyzed using linear regression. (I and J) Relative mRNA level and protein level of MCL1 were determined by RT-qPCR and western blotting in OC cells and human ovarian surface epithelial cells. "P<0.05. SNHG20, small nucleolar RNA host gene 20; MCL1, myeloid cell leukemia 1; WT, wild type; MUT, mutant type; miR-NC, miR-negative control mimic; RT-qPCR, reverse transcription-quantitative PCR; OC, ovarian cancer.

by miR-338-3p inhibitor (Fig. 5D). As shown in Fig. 5E and F, miR-338-3p inhibitor weakened the effect of SNHG20 knockdown on Bcl-2, Bax and c-caspase-3 protein levels. On the other hand, the inhibition effects of SNHG20 knockdown on cell migratory and invasive abilities were blocked by miR-338-3p inhibitor (Fig. 5G and H). Besides, the regulatory effects of SNHG20 knockdown on the levels of EMT markers were weakened by miR-338-3p inhibitor (Fig. 5I and J). Taken together, SNHG20 regulates OC cell progression through suppressing miR-338-3p expression.
SNHG20 upregulates MCL1 level via inhibiting miR-338-3p expression. Using bioinformatics tool starBase v2.0, it was found that MCL1 possessed a complementary sequence with miR-338-3p (Fig. 6A). Dual-luciferase reporter assay showed that the luciferase activity of MCL1 3'UTR-WT, but not MCL1 3'UTR-MUT, was reduced by miR-338-3p, confirming that miR-338-3p interacted with MCL1 (Fig. 6B and C). Moreover, the level of MCL1 was significantly downregulated by miR-338-3p overexpression, which was reversed by SNHG20 upregulation (Fig. 6D and E). These data indicate 

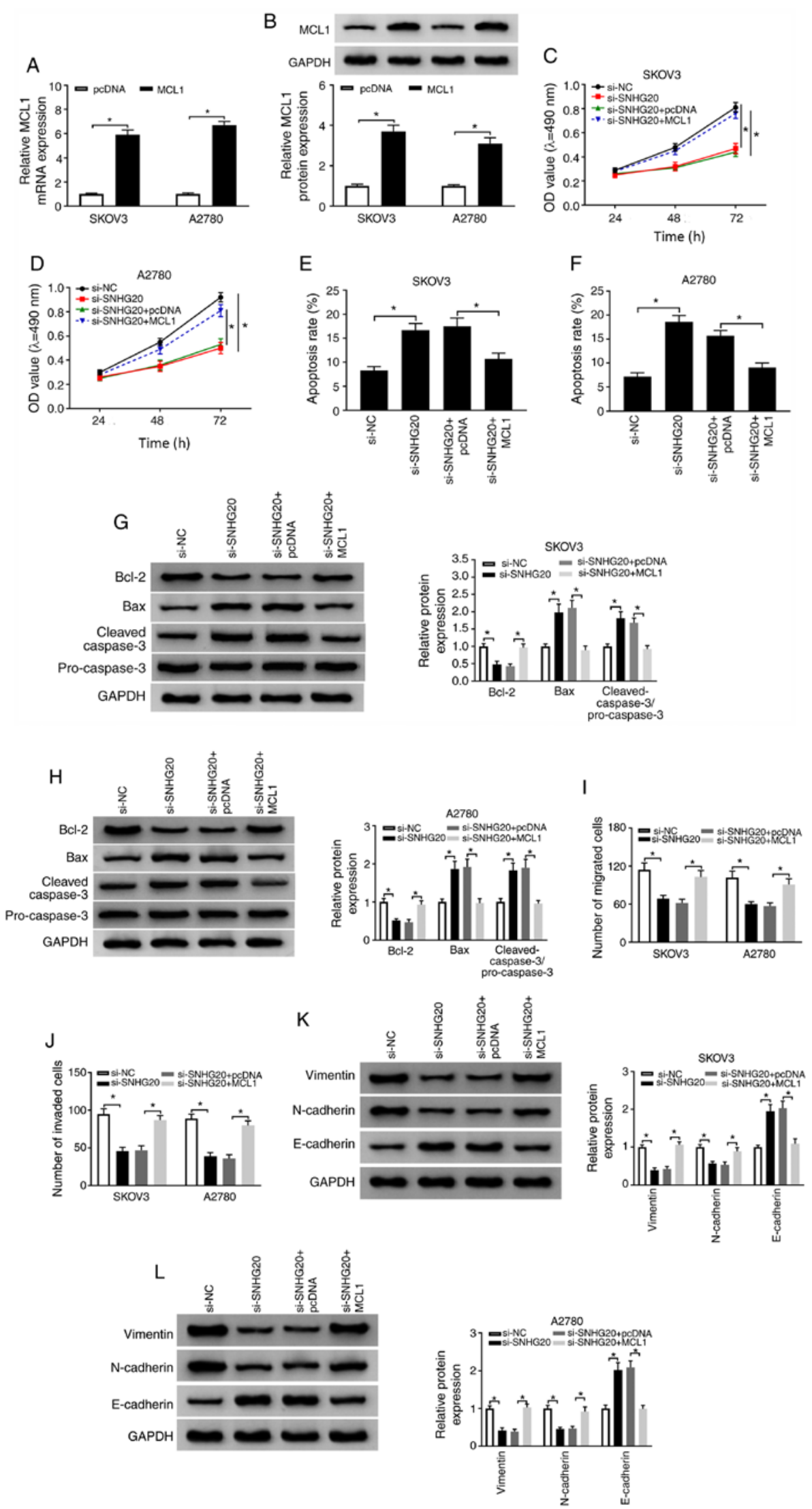

Figure 7. SNHG20 depletion impeded OC progression by regulating MCL1 level. (A and B) Relative mRNA level and protein level of MCL1 were detected by reverse transcription-quantitative PCR and western blotting in SKOV3 and A2780 cells transfected with pcDNA or MCL1. (C-L) SKOV3 and A2780 cells were transfected with si-NC, si-SNHG20, si-SNHG20 + pcDNA or si-SNHG20 + pcDNA-MCL1, respectively. (C and D) MTT assay was used to measure cell proliferation. (E and F) Cell apoptosis rate was detected using flow cytometry. (G and H) Relative levels of cell apoptosis-associated proteins were measured by western blot assay. (I and J) Transwell assay was used to determine cell migratory and invasive abilities. (K and L) Western blot assay was carried out to determine the protein levels of EMT markers. "P<0.05. SNHG20, small nucleolar RNA host gene 20; OC, ovarian cancer; MCL1, myeloid cell leukemia 1; pcDNA, pcDNA3.1 vector; si-NC, small interfering RNA against negative control; si-SNHG20, small interfering RNA against SNHG20; EMT, epithelial-mesenchymal transition. 
A

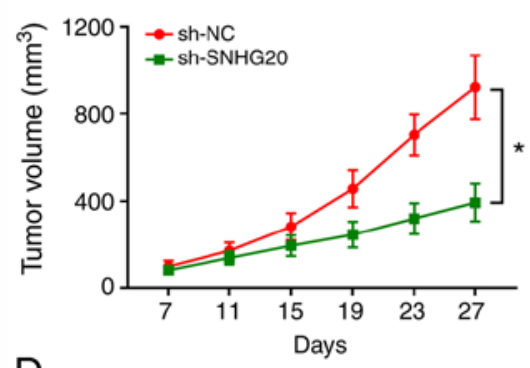

D

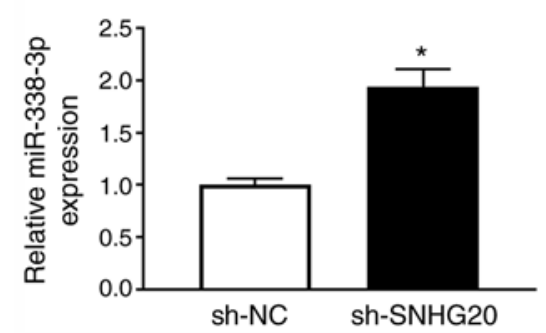

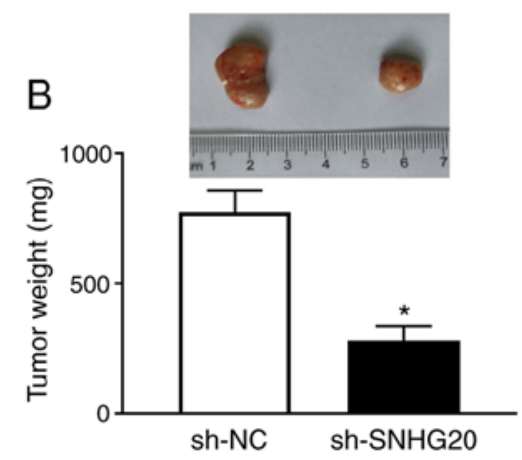

E

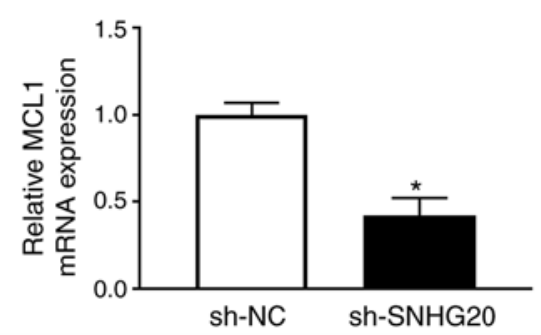

C

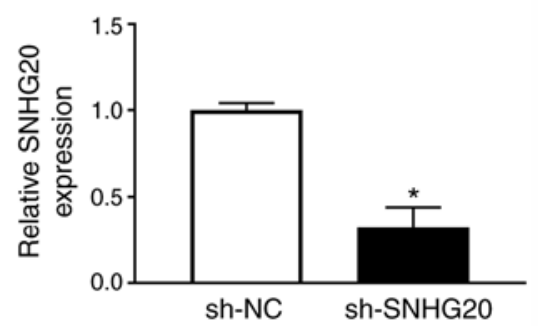

$\mathrm{F}$

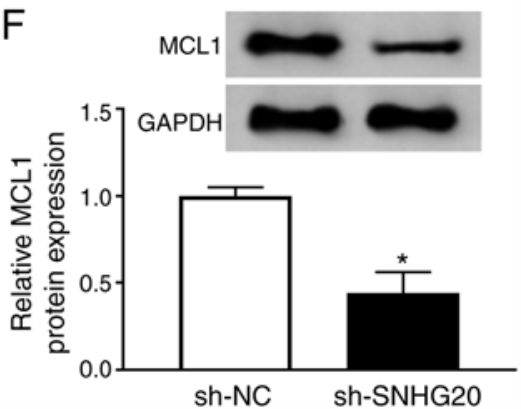

Figure 8. SNHG20 depletion repressed tumor growth in vivo. (A) Tumor volume was calculated in sh-SNHG20 group and sh-NC group every 4 days. (B) Tumor weight was analyzed. (C-E) The levels of SNHG20, miR-338-3p, and MCL1 were detected by reverse transcription-quantitative PCR assay. (F) Relative protein level of MCL1 was determined by western blot assay. "P<0.05. SNHG20, small nucleolar RNA host gene 20; sh-SNHG20, small hairpin RNA against SNHG20; sh-NC, small hairpin RNA against negative control; MCL1, myeloid cell leukemia 1.

that SNHG20 inhibits miR-338-3p expression to increase MCL1 level in OC cells.

Subsequently, the level of MCL1 in OC tissues was detected. The results demonstrated that MCL1 mRNA and protein level were dramatically higher in $\mathrm{OC}$ tissues (Fig. 6F and G). Moreover, MCL1 level was positively correlated with SNHG20 level in OC tissues (Fig. 6H). Besides, increased MCL1 mRNA and protein expression were observed in OC cells (Fig. 6I and J). Thus, MCL1 might act as an oncogene in OC development.

SNHG20 mediates MCL1 expression to regulate OC cell progression. To explore whether SNHG20 exerted its function via regulating MCL1 expression in OC cells, SKOV3 and A2780 cells were transfected with si-NC, si-SNHG20, si-SNHG20 + pcDNA, or si-SNHG20 + MCL1, respectively. RT-qPCR assay confirmed that transfection with MCL1 significantly upregulated MCL1 expression (Fig. 7A and B). As demonstrated in Fig. 7C and D, cell proliferation was suppressed by SNHG20 knockdown, which was rescued by MCL1 overexpression. SNHG20 depletion-induced cell apoptosis was repressed by MCL1 upregulation in OC cells (Fig. 7E and F). Similarly, the effect of SNHG20 knockdown on the levels of cell apoptosis-associated protein was weakened by MCL1 upregulation (Fig. 7G and H). Furthermore, it was found that SNHG20 depletion inhibited cell migration and invasion, while these effects were reversed by MCL1 overexpression (Fig. 7I and J). Besides, MCL1 overexpression weakened the effect of SNHG20 depletion on the levels of EMT markers in OC cells (Fig. 7K and L). These results revealed that SNHG20 promoted OC progression through modulating MCL1 expression.
SNHG20 depletion attenuates tumor growth in vivo. Finally, the effect of SNHG20 on tumor growth was explored in vivo. A2780 cells transfected with sh-SNHG20 was inoculated into the nude mice. The expression levels of SNHG20, miR-338-3p and MCL1 in the A2780 cells transfected sh-SNHG20 are shown in Fig. S1. The data indicated that SNHG20 depletion remarkably repressed tumor volume and weight (Fig. 8A and B). Next, the levels of SNHG20, miR-338-3p, and MCL1 were determined in sh-SNHG20 group and sh-NC group. As expected, SNHG20 level (Fig. 8C) and MCL1 level (Fig. 8E and F) were downregulated, while miR-338-3p level (Fig. 8D) was upregulated in sh-SNHG20 group. Therefore, SNHG20 depletion inhibited tumor growth in vivo.

\section{Discussion}

In recent years, increasing studies indicated that lncRNAs were involved in OC metastasis. For example, 1ncRNA-MALAT1 knockdown repressed cell proliferation and elevated cell apoptosis of OC cells via modulating miR-503-5p level (23). Wang et al (24) demonstrated that CDKN2B-AS1 depletion inhibited OC cell proliferation and mobility through targeting miR-411-3p. You et al (25) demonstrated that DLX6-AS1 upregulation enhanced cell mobility by binding to miR-613 in OC. These data show that lncRNAs play essential roles in OC progression. Therefore, it is essential to explore the role and mechanism of lncRNAs in OC.

SNHG20 was reported as a lncRNA to promote cell development in various human cancer types, including prostate cancer (26), glioma (27), glioblastoma (28), and nasopharyngeal carcinoma (29). In the present study, it was 
found that SNHG20 level was remarkably elevated in OC tissues and cells. This result corroborated with the previous data (30). Previous results indicated that SNHG20 positively regulated OC cell growth (10,31). SNHG20 promoted cell proliferation and migration via silencing the expression of proliferation regulator p21 in non-small cell lung cancer (32). Another study confirmed that SNHG20 promoted gastric cancer progression by inhibiting p21 expression (33). In glioma cells, downregulation of SNHG20 increased cell apoptosis but inhibited cell proliferation by regulating PTEN/PI3K/AKT signaling pathway (34). Moreover, SNHG20 could function as an oncogenic lncRNA by regulating miR-140-5p/ADAM10 axis and MEK/ERK signaling pathway in cervical cancer (35). Furthermore, knockdown of SNHG20 remarkably inhibited cell proliferation, migration and invasion via dysregulating the expression of $\mathrm{p} 21$, cyclin D1, E-cadherin and vimentin in epithelial OC (31). The aforementioned results demonstrated that SNHG20 was closely associated with cancer cell progression. In the present study, it was observed that SNHG20 depletion repressed OC cell proliferation, mobility and promoted apoptosis, suggesting that SNHG20 served as a positive regulator in OC progression.

Present evidence suggested that lncRNA exerted its function through binding to target genes and modulating the levels of targets in cancer (36). For instance, SNHG6 elevated chemoresistance in colorectal cancer cell by targeting miR-26a-5p (37). Bioinformatics tool showed that miR-338-3p is likely to interact with SNHG20. Moreover, SNHG20 negatively regulated the expression of miR-338-3p in OC. Besides, miR-338-3p level was reduced in OC tissues and cells, this result was consistent with the previous data (17). In human cancer, many lncRNAs regulate cancer progression via regulating miR-338-3p expression. For instance, LINC00689 downregulated miR-338-3p level to accelerate glioma development and metastasis (38). LncRNA-SNHG15 repressed miR-338-3p expression to promote colorectal cancer development (39). Furthermore, miR-338-3p was reported to inhibit cell growth in OC $(27,40)$. In the present study, the results suggest that SNHG20 knockdown suppressed OC progression by inhibiting miR-338-3p expression.

In the present study, the data demonstrated that MCL1 was a potential target of miR-338-3p. Furthermore, miR-338-3p was found to reduce MCL1 level in OC cells. Besides, MCL1 was remarkably downregulated in OC tissues and cells. This result was in agreement with the previous reporter (21). MCL1, as an oncogene, positively regulated cell development in many human cancer types, such as gastric cancer (41), lung cancer (42), cervical cancer (43) and osteosarcoma (44). A recent study indicated that MCL1 depletion inhibited cell proliferation and invasion in OC cells (21). The present data indicate that SNHG20 repressed miR-338-3p expression to increase MCL1 level, and MCL1 overexpression weakened the effect of SNHG20 depletion on OC progression. These results suggest that SNHG20 depletion suppressed OC progression via modulating the miR-338-3p/MCL1 axis.

Previous results demonstrated that SNHG20 depletion remarkably repressed tumor growth of OC in vivo (10). The effect of SNHG20 on tumor growth was also investigated in the present study. As expected, tumor volume and weight were significantly suppressed by SNHG20 depletion. Therefore, SNHG20 knockdown attenuated OC tumor growth in vivo.

In conclusion, the present results demonstrate that SNHG20 knockdown represses the development of OC through modulating the miR-338-3p/MCL1 axis, providing a potential target for the therapy of patients with OC.

\section{Acknowledgements}

Not applicable.

\section{Funding}

No funding was received.

\section{Availability of data and materials}

The analyzed data sets generated during the present study are available from the corresponding author on reasonable request.

\section{Authors' contributions}

DW, ZL, HL, JL and QQ made substantial contribution to the concept and design, data analysis, and interpretation of the data; DW, ZL and HL performed the experiments and interpreted the data; DW and QQ drafted the manuscript; DW and JL revised the manuscript critically for important intellectual content. All authors have read and approved the final manuscript.

\section{Ethics approval and consent to participate}

The present study was approved by the ethical review committee of the Affiliated Renhe Hospital of China Three Gorges University. Written informed consent was obtained from all enrolled patients.

\section{Patient consent for publication}

Not applicable.

\section{Competing interests}

The authors declare that they have no competing interests.

\section{References}

1. Siegel RL, Miller KD and Jemal A: Cancer statistics, 2018. CA Cancer J Clin 68: 7-30, 2018.

2. Doubeni CA, Doubeni AR and Myers AE: Diagnosis and management of ovarian cancer. Am Fam Physician 93: 937-944, 2016.

3. Xue Y, Ma G, Gu D, Zhu L, Hua Q, Du M, Chu H, Tong N, Chen J, Zhang $\mathrm{Z}$ and Wang M: Genome-wide analysis of long noncoding RNA signature in human colorectal cancer. Gene 556: 227-234, 2015.

4. Xiao J, Lai H, Wei SH, Ye ZS, Gong FS and Chen LC: lncRNA HOTAIR promotes gastric cancer proliferation and metastasis via targeting miR-126 to active CXCR4 and RhoA signaling pathway. Cancer Med 8: 6768-6779, 2019.

5. Liang H, Su X, Wu Q, Shan H, Lv L, Yu T, Zhao X, Sun J, Yang R, Zhang L, et al: LncRNA promotes ischemic myocardial injury by regulating autophagy through targeting. Autophagy undefined: $1-15,2019$. 
6. Li Q, Zhang J, Su DM, Guan LN, Mu WH, Yu M, Ma X and Yang RJ: lncRNA TUG1 modulates proliferation, apoptosis, invasion, and angiogenesis via targeting miR-29b in trophoblast cells. Hum Genomics 13: 50, 2019.

7. Terashima M, Ishimura A, Wanna-Udom $S$ and Suzuki T: MEG8 long noncoding RNA contributes to epigenetic progression of the epithelial-mesenchymal transition of lung and pancreatic cancer cells. J Biol Chem 293: 18016-18030, 2018.

8. Lu YM, Wang Y, Liu SQ, Zhou MY and Guo YR: Profile and validation of dysregulated long noncoding RNAs and mRNAs in ovarian cancer. Oncol Rep 40: 2964-2976, 2018.

9. Zhang D, Cao C, Liu L and Wu D: Up-regulation of LncRNA SNHG20 predicts poor prognosis in hepatocellular carcinoma. J Cancer 7: 608-617, 2016.

10. He S, Zhao Y, Wang X, Deng Y, Wan Z, Yao S and Shen H: Up-regulation of long non-coding RNA SNHG20 promotes ovarian cancer progression via Wnt/ $\beta$-catenin signaling. Biosci Rep 38: BSR20170681, 2018

11. Trionfini $P$ and Benigni A: MicroRNAs as master regulators of glomerular function in health and disease. J Am Soc Nephrol 28 : 1686-1696, 2017.

12. Wang Y, Kim S and Kim IM: Regulation of metastasis by microRNAs in ovarian cancer. Front Oncol 4: 143, 2014.

13. Sun J, Qiao Y, Song T and Wang H: MiR-495 suppresses cell proliferation by directly targeting HMGA2 in lung cancer. Mol Med Rep 19: 1463-1470, 2019.

14. Liu C, Cai L and Li H: miR185 regulates the growth of osteosarcoma cells via targeting Hexokinase 2. Mol Med Rep 20: 2774-2782, 2019

15. Luo Y,Hao T, Zhang J,Zhang M, Sun P and Wu L: MicroRNA-592 suppresses the malignant phenotypes of thyroid cancer by regulating lncRNA NEAT1 and downregulating NOVA1. Int J Mol Med 44: 1172-1182, 2019.

16. Wang C, Zhang W, Xing S, Wang Z, Wang J and Qu J: MiR-342-3p inhibits cell migration and invasion through suppressing forkhead box protein Q1 in ovarian carcinoma. Anticancer Drugs 30: 917-924, 2019

17. Zhang Y, Shi B, Chen J, Hu L and Zhao C: MiR-338-3p targets pyruvate kinase $\mathrm{M} 2$ and affects cell proliferation and metabolism of ovarian cancer. Am J Transl Res 8: 3266-3273, 2016.

18. Liu X, Wen J, Wang $\mathrm{H}$ and Wang Y: Long non-coding RNA LINC00460 promotes epithelial ovarian cancer progression by regulating microRNA-338-3p. Biomed Pharmacother 108 : 1022-1028, 2018

19. Yip KW and Reed JC: Bcl-2 family proteins and cancer. Oncogene 27: 6398-6406, 2008

20. Zhang S, Zhang M, Jing Y, Yin X, Ma P, Zhang Z, Wang X, Di W and Zhuang G: Deubiquitinase USP13 dictates MCL1 stability and sensitivity to $\mathrm{BH} 3$ mimetic inhibitors. Nat Commun 9: 215, 2018.

21. Su J, Ruan S, Dai S, Mi J, Chen W and Jiang S: NF1 regulates apoptosis in ovarian cancer cells by targeting MCL1 via miR-142-5p. Pharmacogenomics 20: 155-165, 2019.

22. Livak KJ and Schmittgen TD: Analysis of relative gene expression data using real-time quantitative PCR and the 2(-Delta Delta C(T)) method. Methods 25: 402-408, 2001.

23. Sun Q, Li Q and Xie F: LncRNA-MALAT1 regulates proliferation and apoptosis of ovarian cancer cells by targeting miR-503-5p. Onco Targets Ther 12: 6297-6307, 2019.

24. Wang Y,Huang Y, Liu H,Su D, Luo F and Zhou F: Long noncoding RNA CDKN2B-AS1 interacts with miR-411-3p to regulate ovarian cancer in vitro and in vivo through HIF-1a/VEGF/P38 pathway. Biochem Biophys Res Commun 514: 44-50, 2019.

25. You Q, Shi HY, Gong CF, Tian XY and Li S: Long non-coding RNA DLX6-AS1 acts as an oncogene by targeting miR-613 in ovarian cancer. Eur Rev Med Pharmacol Sci 23: 6429-6435, 2019.

26. Wu X, Xiao Y, Zhou Y, Zhou Z and Yan W: lncRNA SNHG20 promotes prostate cancer migration and invasion via targeting the miR-6516-5p/SCGB2A1 axis. Am J Transl Res 11: 5162-5169, 2019.

27. Liu J, Cheng LG and Li HG: LncRNA SNHG20 promoted the proliferation of glioma cells via sponging miR-4486 to regulate the MDM2-p53 pathway. Eur Rev Med Pharmacol Sci 23: $5323-5331,2019$.
28. Gao XF, He HQ, Zhu XB, Xie SL and Cao Y: LncRNA SNHG20 promotes tumorigenesis and cancer stemness in glioblastoma via activating PI3K/Akt/mTOR signaling pathway. Neoplasma 66: 532-542, 2019

29. Sun C, Sun Y and Zhang E: Long non-coding RNA SNHG20 promotes nasopharyngeal carcinoma cell migration and invasion by upregulating TGF- $\beta 1$. Exp Ther Med 16: 4967-4974, 2018.

30. Zhao W, Ma X, Liu L, Chen Q, Liu Z, Zhang Z, Ma S, Wang Z, $\mathrm{Li} \mathrm{H}$, Wang $\mathrm{Z}$ and $\mathrm{Wu}$ J: SNHG20: A vital lncRNA in multiple human cancers. J Cell Physiol: Jan 15, 2019 (Epub ahead of print)

31. Wang D, Dai J, Hou S and Qian Y: LncRNA SNHG20 predicts a poor prognosis and promotes cell progression in epithelial ovarian cancer. Biosci Rep 39: BSR20182186, 2019.

32. Chen Z, Chen X, Chen P, Yu S, Nie F, Lu B, Zhang T, Zhou Y, Chen Q, Wei C, et al: Long non-coding RNA SNHG20 promotes non-small cell lung cancer cell proliferation and migration by epigenetically silencing of P21 expression. Cell Death Dis 8: e3092, 2017.

33. Liu J, Liu L, Wan JX and Song Y: Long noncoding RNA SNHG20 promotes gastric cancer progression by inhibiting p21 expression and regulating the GSK-3 $\beta / \beta$-catenin signaling pathway. Oncotarget 8: 80700-80708, 2017.

34. Guo LP, Zhang ZJ, Li RT, Li HY and Cui YQ: Influences of LncRNA SNHG20 on proliferation and apoptosis of glioma cells through regulating the PTEN/PI3K/AKT signaling pathway. Eur Rev Med Pharmacol Sci 23: 253-261, 2019.

35. Guo H, Yang S, Li S, Yan M, Li L and Zhang H: LncRNA SNHG20 promotes cell proliferation and invasion via miR-140-5p-ADAM10 axis in cervical cancer. Biomed Pharmacother 102: 749-757, 2018.

36. Sun F, Liang W, Tang K, Hong $M$ and Qian J: Profiling the lncRNA-miRNA-mRNA ceRNA network to reveal potential crosstalk between inflammatory bowel disease and colorectal cancer. PeerJ 7: e7451, 2019.

37. Wang X, Lan Z, He J, Lai Q, Yao X, Li Q, Liu Y, Lai H, Gu C, Yan $\mathrm{Q}$, et al: LncRNA SNHG6 promotes chemoresistance through ULK1-induced autophagy by sponging miR-26a-5p in colorectal cancer cells. Cancer Cell Int 19: 234, 2019.

38. Liu X, Zhu Q, Guo Y, Xiao Z, Hu L and Xu Q: LncRNA LINC00689 promotes the growth, metastasis and glycolysis of glioma cells by targeting miR-338-3p/PKM2 axis. Biomed Pharmacother 117: 109069, 2019.

39. Li M, Bian Z, Jin G, Zhang J, Yao S, Feng Y, Wang X, Yin Y, Fei B, You Q and Huang Z: LncRNA-SNHG15 enhances cell proliferation in colorectal cancer by inhibiting miR-338-3p. Cancer Med 8: 2404-2413, 2019.

40. Zhang R, Shi H, Ren F, Liu Z, Ji P, Zhang W and Wang W: Down-regulation of miR-338-3p and Up-regulation of MACC1 indicated poor prognosis of epithelial ovarian cancer patients. J Cancer 10: 1385-1392, 2019.

41. Huang Y, Luo H, Li F, Yang Y, Ou G, Ye X and Li N: LINC00152 down-regulated miR-193a-3p to enhance MCL1 expression and promote gastric cancer cells proliferation. Biosci Rep 38: BSR20171607, 2018

42. Suzuki J, Nakajima W, Suzuki H, Asano Y and Tanaka N: Chaperone-mediated autophagy promotes lung cancer cell survival through selective stabilization of the pro-survival protein, MCL1. Biochem Biophys Res Commun 482: 1334-1340, 2017.

43. Zhou C, Li G, Zhou J, Han N, Liu Z and Yin J: miR-107 activates ATR/Chk1 pathway and suppress cervical cancer invasion by targeting MCL1. PLoS One 9: e111860, 2014.

44. XuW,LiZ,ZhuX,XuRandXu Y:miR-29familyinhibits resistance to methotrexate and promotes cell apoptosis by targeting COL3A1 and MCL1 in osteosarcoma. Med Sci Monit 24: 8812-8821, 2018.

This work is licensed under a Creative Commons

Attribution-NonCommercial-NoDerivatives 4.0 International (CC BY-NC-ND 4.0) License. 